Research Article

\title{
A Mathematical Safety Stock Model for DDMRP Inventory Replenishment
}

\author{
Chan-Ju Lee iD and Suk-Chul Rim \\ Department of Industrial Engineering, Ajou University, Suwon 16499, Republic of Korea \\ Correspondence should be addressed to Chan-Ju Lee; daainlee@hanmail.net
}

Received 15 March 2019; Revised 26 June 2019; Accepted 7 July 2019; Published 19 September 2019

Academic Editor: Alessandro Lo Schiavo

Copyright (c) 2019 Chan-Ju Lee and Suk-Chul Rim. This is an open access article distributed under the Creative Commons Attribution License, which permits unrestricted use, distribution, and reproduction in any medium, provided the original work is properly cited.

\begin{abstract}
The inventory replenishment method of the demand-driven materials requirement planning (DDMRP) draws attention from both scholars and practitioners due to its lower average inventory and, simultaneously, lower stockout rate than existing methods. However, the safety stock of DDMRP replenishment uses a subjective guideline, which is different from any existing safety stock formula. The guideline allows the user to subjectively select the parameter values within a certain range, which jeopardizes the consistency of the inventory performance. This paper proposes an alternative safety stock formula for DDMRP replenishment, which is mathematically defined so as to be consistent. Moreover, simulation indicates that the proposed formula outperforms the DDMRP guidelines and existing safety stock formulas in terms of average inventory and stockout rate.
\end{abstract}

\section{Introduction}

In demand-driven material requirement planning (DDMRP), replenishment is a core concept. DDMRP replenishment approach is different from existing replenishment methods (e.g., Silver et al. [1], Ballou and Srivastava [2], and Brown [3]). The typical research targets in replenishment are to determine when and how much to order. When to order is typically determined as the sum of demand during lead time (DDLT) and safety stock. DDLT is defined as the average demand times average lead time. However, regarding the safety stock, DDMRP replenishment model is very different from that of existing studies.

Most of the inventory replenishment models determine the safety stock level by using the safety factor associated with a given service level, based on the probability theory [1]. However, according to Brown [3], applying a safety stock theory that assumes an incorrect demand distribution shape can cause severe consequences, so it needs to identify the correct distribution shape to determine the safety factor. However, it is difficult to determine the distribution type in practices, since it may vary depending on the span of history data.
As an alternative, Goldratt et al. [4] proposed a replenishment method which does not use the safety factor based on distribution shape. He presented MTA (make-toavailability), which is an inventory replenishment and operation method. Due to its practicality, many successful cases have been reported in the TOCICO Conferences [5]. Ptak and Smith [6] further exploited MTA more specifically in DDMRP. Many successful cases of DDMRP have been reported in the literature (see Section 2.7).

The safety stock of DDMRP replenishment also does not use the safety factor by service level. Instead, DDMRP replenishment guideline [6] allows the users to select the parameter values within a certain range, which will lead to inconsistent results due to its subjectivity. We need an objective formula to determine the safety stock which will obtain more consistent results.

In this study, we propose a new safety stock formula which we derived from the DDMRP replenishment guidelines [6]. The proposed safety stock formula will eliminate subjectivity in determining the safety stock of DDMRP. Moreover, it does not compromise the performance of DDMRP replenishment; rather, better performance is expected in certain situations. 
In order to examine the performance of the proposed safety stock formula, we conduct a simulation of DDMRP replenishment model in which we compare the average inventory and stockout rate of the following three safety stock formulas: DDMRP guideline, existing safety stock formula, and the proposed safety stock formula.

In the following section, we examine the research results related to the safety stock of replenishment models, and subsequently, we review the Theory of Constraints (TOC) replenishment method. In the next subsection, we review the DDMRP replenishment model which is based on the TOC and derive a new safety stock formula. Simulation model and its results are given in the following sections. In the last section, conclusion and future research agenda are presented.

\section{Materials and Methods}

\subsection{Safety Stock of Replenishment Models and DDMRP} Model. We use the following notations in the model:

$$
\begin{aligned}
& d: \text { average daily demand } \\
& S_{d}: \text { standard deviation of daily demand } \\
& L: \text { average replenishment lead time } \\
& S_{L}: \text { standard deviation of replenishment lead time } \\
& z_{\alpha}: \text { safety factor with service level } \alpha \\
& \sigma_{L}: \text { standard deviation of demand during lead time }
\end{aligned}
$$

2.2. Safety Stock Formulas in Existing Models. Brown [3] presented a method to determine the safety stock using the standard deviation of demand during lead time and safety factor as shown in (1). Ballou and Srivastava [2] showed that when $d$ and $L$ are random variables, the standard deviation of demand during lead time is determined as (2):

$$
\begin{aligned}
& \mathrm{SS}=z_{\alpha} \sigma_{L}, \\
& \sigma_{L}=\sqrt{L \cdot s_{d}^{2}+d^{2} \cdot s_{L}^{2}} .
\end{aligned}
$$

The safety factor $z_{\alpha}$ is determined from the service level $\alpha$ which is strategically determined. Recall that, in (1) and (2), both daily demand and replenishment lead time are assumed to follow normal distribution, which we believe is unrealistic. Silver et al. [1] state that "because lead time demand is often skewed right, the normal distribution may not be appropriate." Clark [7] also states that the deviations from normality of the demand during lead time can be characterized almost completely by the skewness. He also states that "in a typical case the optimum value determined a stockout of 2 per cent, but the calculated values would have produced a 7 per cent stockout if skewness had been ignored," indicating that skewness of distribution is also necessary for determining the safety factor in addition to mean and variance. Das [8] states that demand for an item during the delivery lead time can often be represented by a lognormal distribution. Brown [3] also states that exponential distribution and lognormal distribution substantially differ in the expected stockout rates for large safety factor, although the two distributions may look similar. Most existing literature in inventory management considers a certain level of stockout and safety factor based on demand and/or lead time distribution [9]. Since the safety factors differ depending on the distribution shape, the accurate distribution shape must be identified in order to determine an accurate safety factor. However, it is difficult to determine the distribution type in practices, since it may vary depending on the span of history data such as seasonality, or competitors' behavior. Goldratt and Cox, the founder of the Theory of Constraints (TOC) [10], mentioned this point as he proposed the TOC replenishment model in MTA [11].

2.3. TOC Replenishment Model. Goldratt claimed that the stock should be strategically stored in a position within a timeframe of which customers are willing to wait for their orders, and the make-to-availability (MTA) methodology is used for products that must be continuously guaranteed. MTA ensures the complete availability of a certain finished product in a certain warehouse, occupying a selected market [12]. The TOC replenishment method is used in the MTA methodology. Goldratt et al. [4] described this concept in his book "Isn't it obvious," and Schragenheim et al. [11] described specific application methods for the concept in the book "Supply Chain Management at Warp Speed."

The TOC replenishment model is similar to the periodic review model, while the safety stock formula is different. Rather than using a specific formula, it is recommended to determine the safety stock as $50 \%$ of the average demand during lead time and replenishment time, as (3). Note that the safety stock formula of TOC in (3) does not use safety factor, and it does not need to identify the probability distributions:

$$
\mathrm{SS}_{\mathrm{TOC}}=0.5 d(T+L) \text {. }
$$

Goldratt reduced the subjective factor here. Safety stock starts by setting $50 \%$ of the demand during the replenishment lead time regardless of the shape of distribution. And then, according to the trend of demand, the maximum level of stock is adjusted by the given rule instead of the safe stock adjustment. It differs from the existing replenishment in that it focuses on the maximum stock level instead of focusing on accurate safety stock calculations.

In MTA operation, TOC replenishment not only yields good inventory performance, but also focuses on ensuring availability by providing guidance for planning, execution, and control. The effectiveness of MTA method with TOC replenishment has been verified through a series of cases presentations in the TOCICO Conference [5]. One recent example is the milk run replenishment presented by Bonatsos [13] at the TOCICO Conference 2013, in which the average monthly stock decreased, while inventory turnover increased. Han and Kwon [14] compared the forecast-driven approach and TOC replenishment, to conclude that TOC replenishment is superior in terms of stockout, while both methods obtain similar average inventory level.

TOC provides an opportunity to convert inventory management from forecast-driven to demand-driven. 
Christopher stated that most organizations base inventory management on the forecast-driven approach rather than demand-driven approach [15]. He also states that the demand-driven approach is market-sensitive, which means that the supply chain can identify and respond to real demand changes, so it is more effective in maintaining lower inventory than the forecast-driven approach while minimizing stockout. All TOC methods including MTA are based on this demand-driven concept. The demand-driven supply network (DDSN) was defined in AMR research [16]. In addition, Chan et al. [17] studied the demand-driven approach from the perspective of collaborative supply chains. Ptak and Smith [6] reinterpreted the TOC as the demand-driven concept and structured it into the DDMRP.

2.4. DDMRP Components. Ptak and Smith [6] introduced DDMRP as the Third Edition of Orlicky's Material Requirements Planning. Together with Goldratt, Schragenheim and Ptak [18] authored the book "Necessary but Not Sufficient." DDMRP [6] complements the weakness of materials requirement planning (MRP) by integrating the replenishment method with the visibility of the demanddriven method into a single system. Figure 1 shows the components of DDMRP.

The first stage in Figure 1, strategic inventory positioning, is the stage in which inventory position is determined. In DDMRP, inventory positioning is very important. On the Bill of Material (BOM), the strategically determined stock item is referred to as the decoupling point. At this decoupling point, stock is replenished. As customers require shorter lead time, it directly affects the stock level. Since variability increases for longer lead time, the stock of appropriate items is strategically placed, based on the BOM, thereby decoupling the linked variability. Since the end item is produced using parts and components in stock, the lead time offered to customers can be shortened.

In the second stage, buffer profiles and levels, the buffer levels are determined and the maximum level of stock $(M)$ is defined. In the third stage, like the TOC replenishment method, buffer levels are adjusted when item characteristics change. In the fourth stage, the replenishment amount is calculated. The fifth stage, visible and collaborative execution, is the control step similar to the TOC replenishment method.

2.5. DDMRP Replenishment and Its Guideline. The DDMRP replenishment model is similar to the min-max model. In the DDMRP replenishment model, a maximum level of stock $(M)$ is expressed as the sum of three components: yellow zone (YZ), red zone (RZ), and green zone (GZ). YZ is defined as average daily demand multiplied by average lead time, as in (4), so it corresponds to the demand during lead time in the existing methods. RZ represents the safety stock in the existing replenishment models. Note that, in the reorder point model (or min-max model), an order is placed when the inventory position falls below the reorder point, which is the sum of DDLT and safety stock. Likewise, in DDMRP replenishment, an order is placed when the

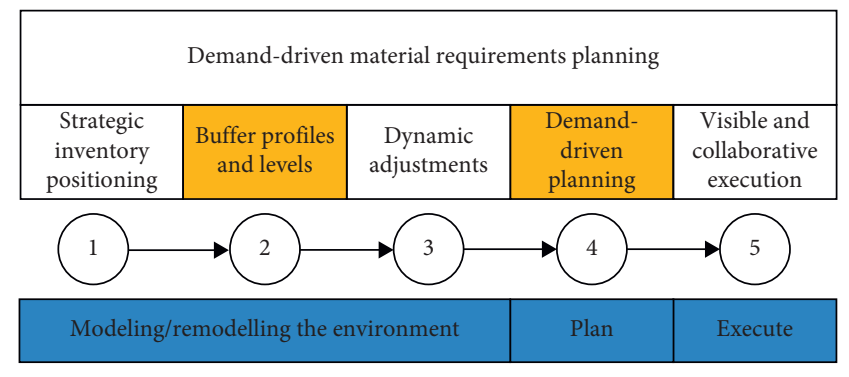

Figure 1: DDMRP components [6].

inventory position falls below the top of yellow, which is the sum of DDLT (i.e., YZ) and RZ, as in (10). So RZ clearly corresponds to the safety stock in the existing replenishment models.

$\mathrm{RZ}$ is expressed as the sum of red zone base (RB) and red zone safety (RS) as in (5). RB is defined as YZ multiplied by the lead time factor in Table 1, as in (6). RS is defined as RB multiplied by the variability factor in Table 2, as in (7). GZ represents the order quantity and defined as YZ multiplied by the lead time factor in Table 1, as in (8). Top of green (TOG) is the sum of YZ, RZ, and GZ, as in (9), and represents the maximum stock level $(M)$ in the existing replenishment models. Top of yellow (TOY) is the sum of YZ and RZ, as in (10), and represents the reorder point in the existing models:

$$
\begin{aligned}
\mathrm{YZ} & =d \cdot L, \\
\mathrm{RZ} & =\mathrm{RB}+\mathrm{RS}, \\
\mathrm{RB} & =\mathrm{YZ} \cdot F_{L}, \\
\mathrm{RS} & =\mathrm{RB} \cdot F_{V}, \\
\mathrm{GZ} & =(d \cdot L) \cdot F_{L}, \\
\mathrm{TOG} & =\mathrm{GZ}+\mathrm{YZ}+\mathrm{RZ}, \\
\mathrm{TOY} & =\mathrm{YZ}+\mathrm{RZ} .
\end{aligned}
$$

The lead time factor $\left(F_{L}\right)$ and variability factor $\left(F_{V}\right)$ in (6) and (7) can be determined by the user using the guidelines [19] shown in Tables 1 and 2, respectively. Note that RS in (7) does not explicitly consider the lead time variability. The guideline [6] is presented for three relative variability levels (high, medium, or low); three relative length of lead time (long, medium, or short); three item types (make, purchased, or distributed); and two types of order quantities (minimum order quantity or not), total of 54 cases. Among those, for items to buy, the guideline $[6,19]$ presented the lead time factor as in Table 1.

2.6. Order Spike and Replenishment Quantity in DDMRP. To address the replenishment quantity (RQ) calculation in DDMRP, we first need to address the order spike. The order spike is a qualifying quantity of known cumulative demand 
TABle 1: Guideline for lead time factor $F_{L}$ [19].

\begin{tabular}{lcc}
\hline Lead time & $F_{L}(\%)$ & Purchased part (days) \\
\hline Long & $20-40$ & $26+$ \\
Medium & $41-60$ & $11-25$ \\
Short & $61-100$ & $1-10$ \\
\hline
\end{tabular}

TABLe 2: Guideline for variability factor $F_{V}$ [19].

\begin{tabular}{lc}
\hline Variation & $F_{V}(\%)$ \\
\hline High & $61-100$ \\
Medium & $41-60$ \\
Low & $20-40$ \\
\hline
\end{tabular}

within a qualifying time window that threatens the integrity of the buffer [19]. That means that the qualifying level, namely, the order spike threshold (OST), and the qualifying time window, namely, the order spike horizon (OSH) must be defined [6]. If the total orders received for an item on a specific date are greater than the threshold, then the amount is regarded as the order spike, as shown on the fourth day in Figure 2. Note that the cumulated order on day 9 is not regarded as an order spike since it is currently out of the order spike horizon (5 days). OSH is typically set to be the replenishment lead time. Ptak and Smith [6] recommend OST value to be determined as $50 \%$ of RZ.

With order spikes, replenishment is requested in advance for the daily lumpy demand with confirmed order, allowing earlier response to the lumpy demand. This results in a substantial reduction of stockout. Clark [7] explains this as follows: "The distribution of daily demand was not only skewed but even bimodal. The demand during lead time is characterized by three parameters, mean, standard deviation, and the coefficient of skewness." Safety stock that does not reflect skewness cannot respond to lumpy demand; however, the order spike concept can effectively respond to lumpy demand without considering skewness.

In DDMRP replenishment, order is placed when the available stock is below TOY. However, there is a difference in the method of determining the replenishment quantity. Generally, the replenishment quantity is the maximum stock minus available stock. Recall that Brown [20] defines available stock (AS) as on-hand $(H)$ plus schedule receipt $(R)$ minus allocated quantity $(A)$, as in (11). However, in DDMRP, the replenishment order quantity (RQ) is defined as top of green (TOG) minus available stock (AS) plus order spike quantity $(k)$, as in (12):

$$
\begin{aligned}
& \mathrm{AS}=H+R-A, \\
& \mathrm{RQ}=\mathrm{TOG}-\mathrm{AS}+k .
\end{aligned}
$$

2.7. DDMRP-Related Research. Success stories from companies that have applied the DDMRP method have been continuously announced. According to the Demand Driven Institute [21], improved performance of numerous enterprises, such as Royal Engineered Composites, Figeac Aero, Allergan, Stemcell Technologies, Michelin, MIC, Oregon

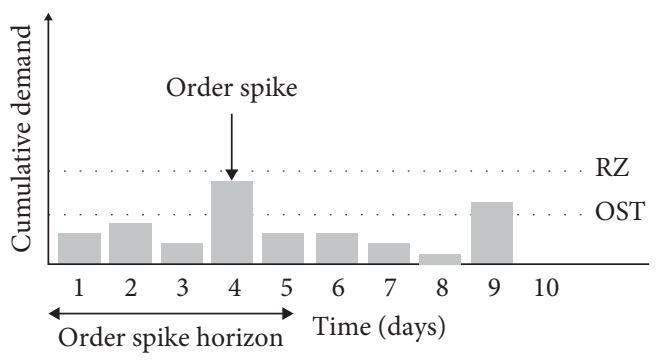

Figure 2: An example of order spike on day 4.

Freeze Dry, and PZ Cussons, is evidence of the high practicality of this method.

While there have been many DDMRP-related studies, most of them compare with MRP. Lee and Jang [22] demonstrated DDMRP's superiority through a system dynamic simulation with DDMRP and MRP. Ihme [23] applied DDMRP to a printer ink manufacturer, demonstrating improved performance, in which the stock level and stockout are reduced by approximately $45 \%$ and $95 \%$, respectively. Miclo et al. [24] used a discrete-event simulation approach to conduct an objective and quantitative comparison between DDMRP and MRP. Shofa and Widyarto [25] presented a paper studying MRP and DDMRP to effectively control production in the automobile industry. Similar to these examples, most of the other studies were comparisons of MRP and DDMRP. Other than these examples, Rim et al. [26] developed an algorithm to optimize strategic inventory position in BOM. Our study is unique in that we present a mathematical approach to determine the red zone level for the DDMRP replenishment, which has not been reported in the literature.

2.8. Excessive Deviation of the Safety Stock in DDMRP. In DDMRP guideline for the safety stock (RZ), the lead time factor and variability factor are selected arbitrarily within a certain range given in Tables 1 and 2. The critical weakness of DDMRP replenishment method is that excessively large deviation can be obtained by subjective choice of the factors, so that the performance will be naturally inconsistent. For example, consider the average demand of 1,000 units and the average lead time of 5 days. The guideline from Table 1 will offer the user to choose the lead time factor between 0.61 and 1.0 , and you may choose the variability factor between 0.2 and 1.0 if no information is available about the demand variability. Recall that, from (5) to (7), $\mathrm{RZ}=\mathrm{RB}+\mathrm{RS}=d L F_{L}\left(1+F_{V}\right)$. So the maximum safety stock will be determined as $1,000(5)(1.00)(1+1.00)=10,000$, while minimum as $1,000(0.61)(1+0.20)=3,660$. Safety stock can be determined as three times larger than the minimum, which is excessively inconsistent. For medium and long lead time cases, the gap between minimum and maximum is about 2.4 time or 3.3 times greater than safety stock level, respectively, as shown in Table 3.

Such an excessive inconsistency of the existing guideline for safety stock level in [6] is the core motivation of this study. In the following section, we will present an alternative 
TABLE 3: Safety stock level obtained from the various parameter values.

\begin{tabular}{|c|c|c|c|c|c|c|c|c|c|}
\hline & & & & & & & & & \\
\hline & & & & & & & & & \\
\hline & & & & Min & Max & Min & Max & Min & Max \\
\hline Lead & & & & 0.20 & 0.40 & 0.41 & 0.60 & 0.61 & 1.00 \\
\hline Iong & 35 & Min & 0.20 & 8,400 & & 9,870 & & 11,270 & \\
\hline Long & 35 & Max & 0.40 & & 19,600 & & 22,400 & & 28,000 \\
\hline & & Min & 0.41 & 9,840 & & 11,562 & & 13,202 & \\
\hline Medium & 20 & Max & 0.60 & & 16,800 & & 19,200 & & 24,000 \\
\hline Short & 5 & Min & 0.61 & 3,660 & & 4,301 & & 4,911 & \\
\hline Short & 5 & Max & 1.00 & & 7,000 & & 8,000 & & 10,000 \\
\hline
\end{tabular}

${ }^{*}$ Empty cells indicate irrelevant combinations.

approach to determine the safety stock level of DDMRP replenishment, which will result in more consistent and better performance in terms of average inventory and shortage.

\section{Proposed Safety Stock Model in DDMRP Replenishment}

In this paper, we propose a new safety stock (RZ) formula, which will mitigate the vulnerability of RZ of DDMRP replenishment, based on the following direction: first, the formula is still based on the DDMRP guidelines, but avoids inconsistency due to subjective choice of values within a range. Second, parameter values must be obtainable from the historical data. Third, both demand variability and lead time variability are considered. Fourth, safety factor based on the service level is not used. Fifth, according to the demanddriven concept, shortage must be minimized, while average inventory not be increased. Since RZ comprises the two components RB and RS, based on the above direction, we will propose the modified $\mathrm{RB}_{p}$ and $\mathrm{RS}_{p}$, respectively, as follows.

3.1. Proposed $R B_{p}$. Recall that, from (4) and (6), the existing $\mathrm{RB}$ is defined as in (13). Ptak and Smith [19] allow a subjective choice of a value $F_{L}$ within the range given in Table 1 to determine $\mathrm{RB}$ :

$$
\mathrm{RB}=d L F_{L}
$$

In Table 1, it is implied that for shorter lead time case, the larger portion of DDLT and for the longer lead time case the smaller portion of DDLT be used as RB. Instead of such a subjective and qualitative relation, we propose an alternative quantitative expression of inverse relation that $F_{L}$ is expressed as $1 / \sqrt{L}$. Then, the resulting $R B_{1}$ is expressed as shown in the following equation:

$$
\mathrm{RB}_{1}=d L(1 / \sqrt{L})=d \sqrt{L}
$$

Now, in Figure 3, the highest plotting curve represents $f_{1}(L)=L F_{L}$, where $F_{L}$ is computed by the linear interpolation for the three ranges given in Table 1. Although the long lead time in Table 1 is defined as over 25 days, for linear interpolation, we need to set the upper limit of the range. We consider 90 days as the longest lead time since most imported make-to-order (MTO) items often take about three months. The lowest curve represents $f_{2}(L)=\sqrt{L}$ as in (14). It is clearly observed that the two curves differ significantly. From the simulation in our earlier study [27], we observed that DDMRP guideline yields excessive inventory for long lead time and some shortage for shorter lead time and higher variability. We also observed that $f_{2}(L)$ is too low to avoid shortage [27].

Hence, we adjust $f_{2}(L)$ to devise an alternative function $f_{3}(L)$ in such a way that $f_{3}(L)$ is $f_{2}(L)$ amplified by $\beta$ for $0<\beta<2$ and shifted by $\gamma$ for $0<\gamma<10$, as shown in the following equation:

$$
\mathrm{RB}_{p}=d(\beta \sqrt{L}+\gamma) .
$$

The performance of $\mathrm{RB}_{p}$ in (15) will depend on the parameter values $\beta$ and $\gamma$. So in order to find the pair of $\beta$ and $\gamma$ that yields the best performance, that is, the lowest average inventory with no shortage, for lead time of 5, 20, 35, and 60 days, $\mathrm{CV}_{d}=0.7$ and $\mathrm{CV}_{L}=0.4$, we perform a simulation for the range $0<\beta<2$ and $0<\gamma<10$, to obtain $\beta^{*}$ and $\gamma^{*}$ to be 1.02 and 1.15 , respectively. The solid line in Figure 3 shows $f_{3}(L)$. Therefore, we propose $\mathrm{RB}_{p}$ as

$$
\mathrm{RB}_{p}=d(1.02 \sqrt{L}+1.15) \text {. }
$$

3.2. Proposed $R S_{p}$. Recall that DDMRP defined the safety stock level as the sum of the two terms, one is RB which considers lead time factor and the other is RS which considers demand variability. That is, DDMRP's safety stock does not explicitly consider lead time variability. Hence, we want an alternative expression for RS to be the one which considers both demand variability and lead time variability. This naturally leads the authors to consider $\sigma_{L}$, the existing standard deviation of the demand during lead time when both demand and lead time varies, as in (2). So, we propose RS to be equal to $\sigma_{L}$. Then (2) can be rewritten as (17) (recall that the coefficient of variation $(\mathrm{CV})$ is defined as $\mathrm{CV}=\sigma / \mu)$ :

$$
\begin{aligned}
\sigma_{L} & =\sqrt{L \cdot s_{d}^{2}+d^{2} \cdot s_{L}^{2}} \\
& =d \cdot \sqrt{L} \cdot \sqrt{\mathrm{CV}_{d}^{2}+L \cdot \mathrm{CV}_{L}^{2}} .
\end{aligned}
$$

From (14), (17) can be rewritten as follows: 


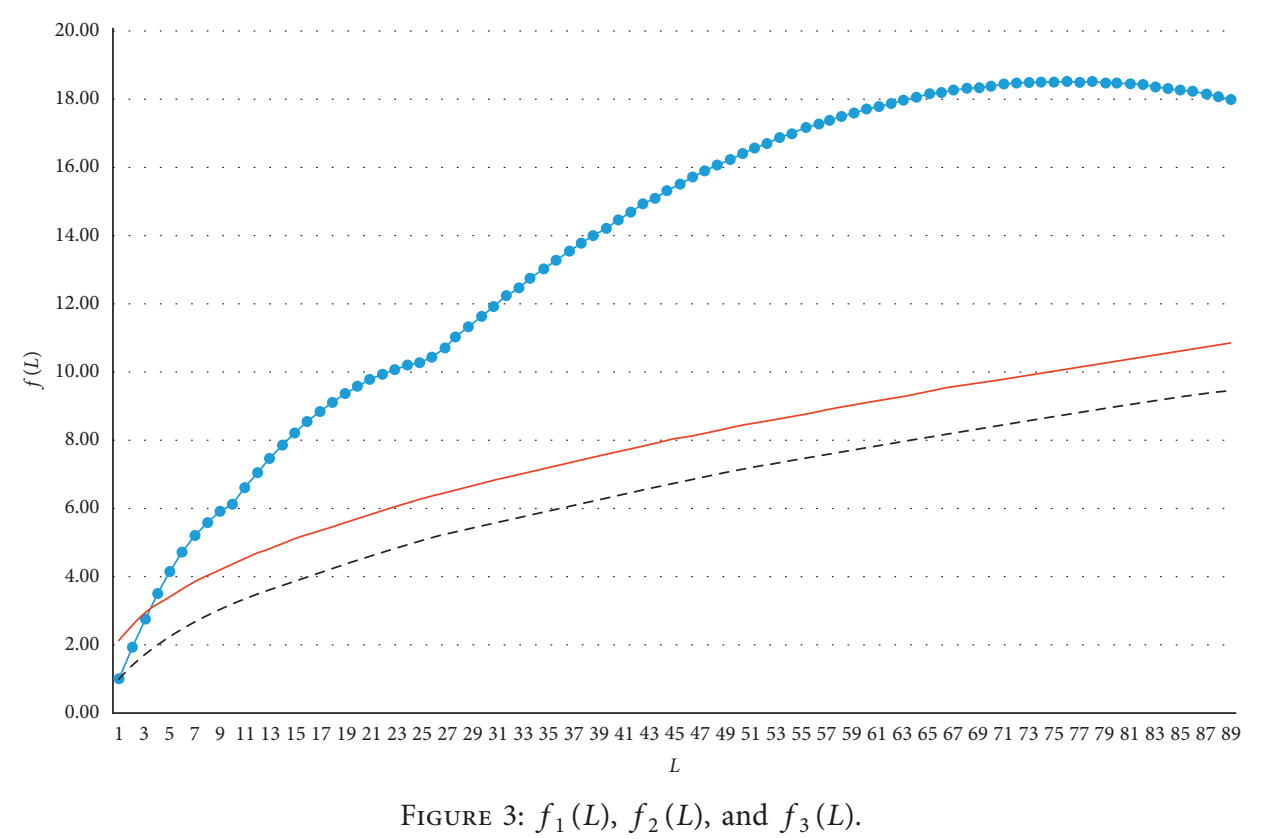

$$
\mathrm{RS}_{1}=\mathrm{RB}_{1} \sqrt{\mathrm{CV}_{d}^{2}+\mathrm{CV}_{L}^{2} \cdot L} .
$$

Unlike $F_{V}$ in (7), $\sqrt{\mathrm{CV}_{d}^{2}+\mathrm{CV}_{L}^{2} \cdot L}$ is a factor that considers both demand variability and lead time variability. Using the adjusted $\mathrm{RB}_{p}$ in (16), $\mathrm{RS}_{p}$ is written as follows:

$$
\begin{aligned}
\mathrm{RS}_{p} & =\mathrm{RB}_{p} \sqrt{\mathrm{CV}_{d}^{2}+\mathrm{CV}_{L}^{2} \cdot L} \\
& =d(\beta \sqrt{L}+\gamma) \sqrt{\mathrm{CV}_{d}^{2}+\mathrm{CV}_{L}^{2} \cdot L} .
\end{aligned}
$$

Consequently, from (16) and (19), we propose (20) as an alternative quantitative formula to the safety stock of DDMRP replenishment model:

$$
\begin{aligned}
\mathrm{RZ}_{p} & =\mathrm{RB}_{p}+\mathrm{RS}_{p} \\
& =[d(1.02 \sqrt{L}+1.15)]\left[1+\sqrt{\mathrm{CV}_{d}^{2}+\mathrm{CV}_{L}^{2} \cdot L}\right] .
\end{aligned}
$$

Now the proposed quantitative model does not use $F_{L}$ and $F_{V}$ from the guideline, so it is free from arbitrariness and will yield a consistent result. In the next section, we will examine the average performance of the proposed safety stock formula (20) against the DDMRP guideline and existing safety stock formula using simulation.

\section{Simulation}

In order to evaluate the average performance of the proposed safety stock level given in (20), we conduct a simulation using Excel. We compare three safety stock models: the existing DDMRP safety stock (E-SS) shown in (5), traditional safety stock (T-SS) shown in (1), and the proposed safety stock (P-SS) shown in (20). We assume that backorder is accepted. Note that the three models differ only in determining its safety stock level, and replenishment methods are the same, that is, place a replenishment order when the available stock level falls below the reorder point (which is TOY for E-SS and P-SS, and $d L+z_{\alpha} \sigma_{L}$ for T-SS), and order quantity includes order spike as in (12). For each model, we repeat simulation for 10 times, with each run length of 365 days.

4.1. Parameters. We consider the following cases: (1) three average lead times $(5,20$, and 35 days) which correspond to short, medium, and long lead time, respectively; (2) six coefficient of variation values of lead time $(0.00,0.02,0.04$, $0.06,0.08$, and 010 ); and (3) three coefficient of variation values of demand $(0.3,0.5$, and 0.7$)$, which correspond to low, medium, and high demand variability, respectively. A total of 54 cases are simulated. For E-SS, using linear interpolation from Table 1 , the lead time factors $\left(F_{L}\right)$ are obtained to be $82.7 \%, 47.8 \%$, and $37.2 \%$ for 5,20 , and 35 days of lead time, respectively. For T-SS, safety factor $z_{\alpha}$ is selected as 5.0 to ensure "close to zero" shortage.

We use average daily demand of 1,000 units. Ptak suggested that minimum order quantity (MOQ) be used if it is smaller than the order quantity in (12). In this simulation, we use MOQ of 2,000 units as the order quantity.

Regarding the distribution of the daily demand and replenishment lead time, in order to simulate the proposed model using parameters that are close to the actual data in reality, we select lognormal distribution as the appropriate realistic distribution based on the following statements: Silver et al. [1] state that because lead time demand is often skewed right, the normal distribution may not be an appropriate; Das [8] states that demand for an item during the delivery lead time can often be represented by a lognormal distribution; and Tadikamalla [28] states that the percentiles and cumulative distribution function values of the lognormal distribution can easily be calculated from the corresponding standard normal values.

To generate random daily demand and lead time data following lognormal distribution, the Excel LOGINV function is used as in (21) and (22), respectively: 


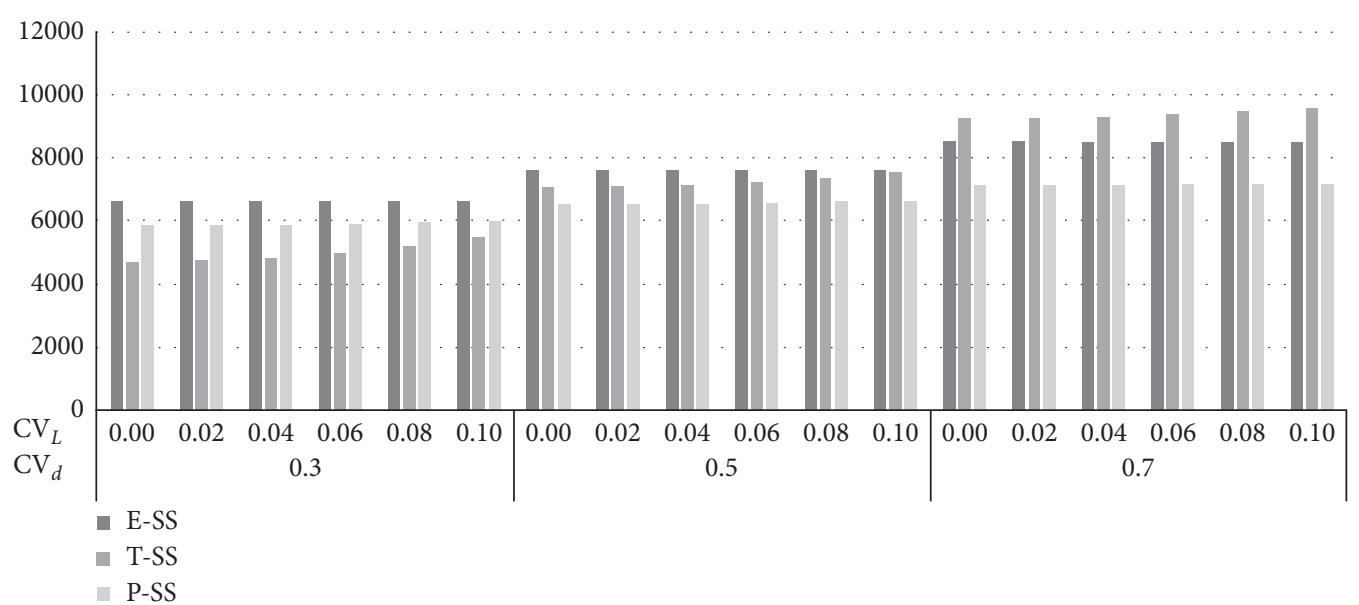

FIgURE 4: Average inventory for $L=5$.

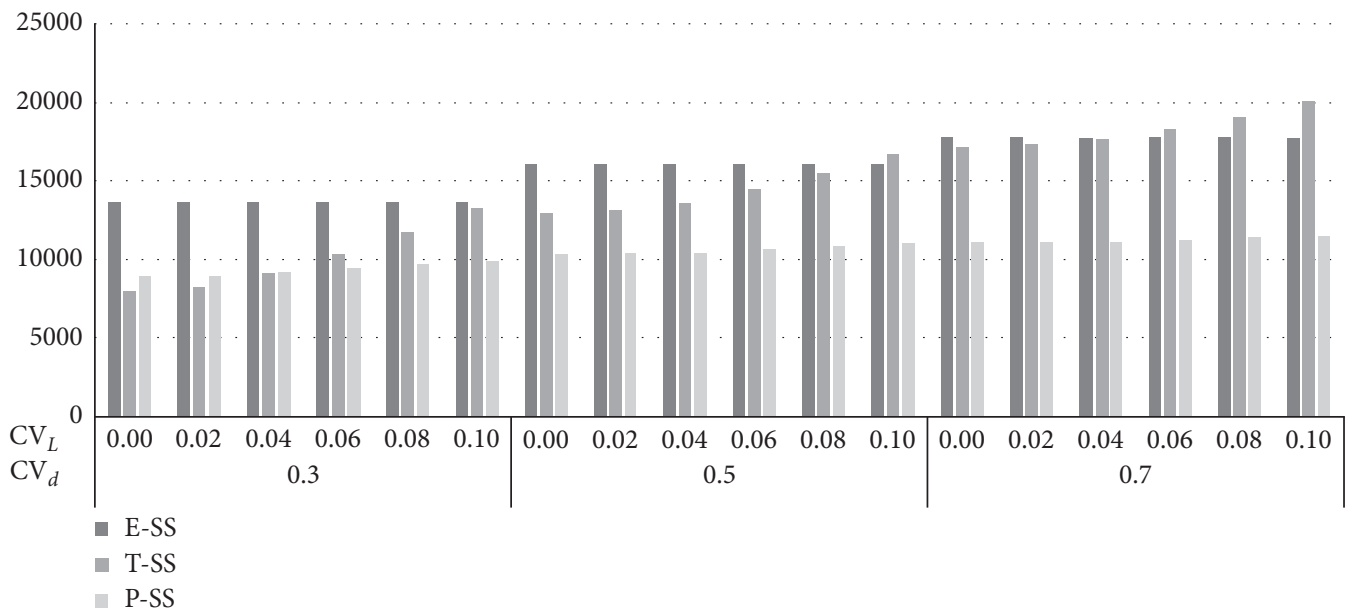

Figure 5: Average inventory for $L=20$.

Daily demand $=\operatorname{ROUND}\left(\operatorname{LOGINV}\left(\operatorname{RAND}(), D_{1}, D_{2}\right), 0\right)$,

where $D_{1}=\mathrm{LN}(d)-D_{2}^{2} / 2, D_{2}=\sqrt{\operatorname{LN}\left(S_{d}^{2} /\left(d^{2}+1\right)\right)}$.

Lead time $=\operatorname{ROUND}\left(\operatorname{LOGINV}\left(\operatorname{RAND}(), L_{1}, L_{2}\right), 0\right)$,

where $L_{1}=\mathrm{LN}(L)-L_{2}^{2} / 2, L_{2}=\sqrt{\operatorname{LN}\left(S_{L}^{2} /\left(L^{2}+1\right)\right)}$.

\section{Results and Discussion}

The performance of the inventory management method is typically measured by the average inventory level and shortage rate. Since DDMRP replenishment model is known to yield "close to zero" shortage, we set safety factor $z_{\alpha}$ as 5.0 for T-SS to ensure "close to zero" shortage and to compare the average inventory level among the three models, as shown in the following figures for $L=5,20$, and 35 , respectively.

Note that the average inventory of E-SS remains unchanged as CVL varies, since DDMRP model does not consider lead time variability, although it considers the relative magnitude of lead time as long, medium, or short, as in Table 1. It is observed in Figure 4 that the average inventory of the proposed model P-SS is lower than E-SS in all cases and lower than T-SS when $\mathrm{CV}_{d}=0.5$ or 0.7 .

For medium lead time of 20 days, Figure 5 shows that the average inventory level of P-SS is significantly lower than E-SS in all cases and lower than T-SS in all cases except when $\mathrm{CV}_{d}=0.3$ and $\mathrm{CV}_{L}$ is very small. Note that the average inventory of T-SS substantially increases as $\mathrm{CV}_{d}$ increases, while that of P-SS increases by a small magnitude.

For long lead time of 35 days, Figure 6 shows that the average inventory level of P-SS is far lower than E-SS in all cases and lower than T-SS in all cases except when $\mathrm{CV}_{d}=0.3$ and $C_{L}$ is very small. Recall that zero shortage is ensured in all cases.

Consequently, simulation results indicate that the safety stock model that we proposed in (20) significantly outperforms the existing DDMRP replenishment method for all cases and significantly also outperforms the traditional safety stock model for almost all cases, under the same "zero shortage" situation. Hence, we believe that the proposed safety stock formula will provide more consistent results and, therefore, can be effectively used in practices. 


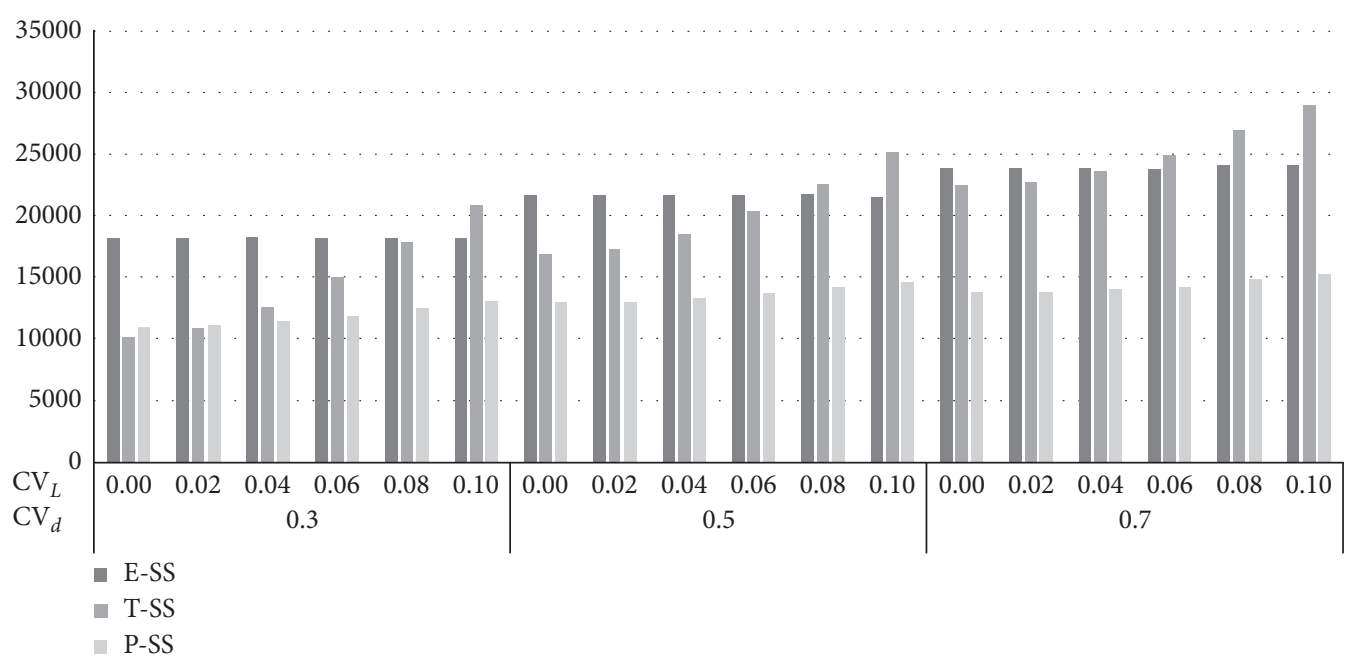

Figure 6: Average inventory for $L=35$.

The above simulation result is for $0 \leq \mathrm{CV}_{L} \leq 0.1$, which we believe sufficiently covers practical uncertainty in today's business. However, for academic curiosity, we have conducted further simulation for $\mathrm{CV}_{L}=0.2,0.3$, and 0.4 , as it may bring more insights to the validity of the proposed formula. For $\mathrm{CV}_{L}=0.4$, shortage occurs 5 times in E-SS, while only one time in P-SS. It is observed that the average inventory of P-SS is higher than that of E-SS only when lead time $=5$, and $\mathrm{CV}_{L}=0.3$ and $0.4, \mathrm{CV}_{d}=0.3$ and 0.5 ; or $\mathrm{CV}_{L}=0.4, \mathrm{CV}_{d}=0.7$. That is, it turns out that the proposed model outperforms the existing DDMRP replenishment model in most of the cases for higher demand variability as well.

\section{Conclusion}

In this paper, we propose an alternative model to determine the safety stock level for the existing DDMRP inventory replenishment model. The motivation of this study is twofold. Firstly, the existing guideline to determine the safety stock level in DDMRP lacks consistency as it allows the users to select values subjectively from a range. Secondly, the existing DDMRP replenishment yields excessively high inventory level in the practical parameter value range, although it yields lower inventory than the traditional safety stock formula for some cases.

Hence, we propose a method to mathematically determine the safety stock level for DDMRP, which will yield lower inventory level than the existing DDMRP guideline, while still maintaining the shortage rate close to zero. The proposed model basically follows the frame of the existing DDMRP inventory replenishment model, in which the safety stock comprises red zone base (RB) and red zone safety (RS). Instead of using subjective guideline given in Tables 1 and 2, we propose an alternative mathematical model for RB and RS.

The proposed RB reflects the observation that the existing guideline yields excessive inventory for longer lead time and some shortage for shorter lead time. Within the valid range of parameter values that will yield better performance than the existing guideline, we identified the best modifying parameters $\beta$ and $\gamma$ for the formula using simulation for one year for ten times. The proposed RS is devised in that not only demand variability but also lead time variability is mathematically considered.

We have compared using simulation the average inventory level of the proposed safety stock formula against the existing DDMRP guideline and traditional safety stock formula over the range of practical parameter values. Skewness of demand and lead time distribution has been considered by using lognormal distribution since we believe it is more realistic.

Under "no shortage" situation, the average inventory of the proposed formula outperforms the existing guideline for all cases and outperforms traditional safety stock formula for all cases except when the demand variability is small. Hence, we conclude that the proposed safety stock formula provides more consistent results and, therefore, can be effectively used in practices.

Recall that the existing inventory replenishment models require a certain demand and/or lead time distribution in order to determine the optimal safety factor (i.e., service level) to minimize the sum of inventory and shortage cost. However, the proposed model does not use the safety factor for a specific demand and/or lead time distributions. Therefore, our proposed model can be regarded as more practical as it is simpler than the existing models and yet outperforms the existing models in terms of average inventory level.

For further study, we suggest that alternative distributions for demand and/or lead time such as Gamma and Weibull distribution be used for simulation. We also suggest that simulation expands the range of the parameter values including the coefficient of variation of demand beyond 0.7 up to 1.0 .

\section{Data Availability}

The Excel data used to support the findings of this study are included within the supplementary information file. 


\section{Conflicts of Interest}

The authors declare that they have no conflicts of interest.

\section{Acknowledgments}

TOC VISION, a consulting firm in South Korea, has partially supported this research.

\section{Supplementary Materials}

Supplementary materials contain three excel files, which are as follows: (1) 6496309-simulation-LT CV 000-002004.xlsm, (2) 6496309-simulation-LT CV 006-008-010.xlsm, and (3) 6496309-simulation result-summary.xlsm. The files (1) and (2) have simulation logic. File (1) is the simulation for lead time coefficient of variation $(\mathrm{CV})$ of $0.00,0.02$, and 0.04 , and File (2) is the simulation for $\mathrm{CV}=0.06,0.08$, and 0.10 . All other contents are the same. There are five sheets in each of the two Excel files. The first sheet generates demand and lead time data. The second E-SS sheet is the simulation results using the existing DDMRP safety stock formula; the third T-SS is the simulation results using the traditional safety stock formula; the fourth P-SS sheet is the simulation results using the proposed safety stock formula; and the fifth is the result comparison sheet, which compares the results of the three simulations. 1-1: sheet data in two files. The data sheet generates random daily demand and lead time. Daily demand is generated using equation (21) with average of 1,000 and CV of high, medium, and low. The daily lead time is generated using equation (22) for the combinations of lead time (long, medium, and short) and lead time CV. Three simulations are conducted using the same parameter values for fair comparison. 1-2: sheets E-SS, T-SS, and P-SS. Each of the three simulation sheets has the same format. We used functions and Macro programs in the simulation. Row 1: from $\mathrm{R}$ to $\mathrm{Z}$ are Macro Buttons. When you click each button, the rows of the corresponding columns are stored in the corresponding rows (5 to 14 ) in column $\mathrm{H}$. Rows 2 4: columns $\mathrm{R}$ to $\mathrm{Z}$ are the standard deviation of lead time. Row 6: columns $\mathrm{R}$ to $\mathrm{Z}$ are the $\mathrm{CV}$ of lead time. Rows 7 9: column $\mathrm{R}$ is demand during lead time (yellow zone); column $\mathrm{S}$ calculates red zone base using formula (6) for E-SS and (16) for P-SS, and cell $(7, \mathrm{~T})$, cell $(7, \mathrm{~W})$, and cell $(7, \mathrm{Z})$ are the CV of demand; cell $(8, T)$, cell $(8, W)$, and cell $(8, Z)$ are daily average demand; cell $(9, \mathrm{~T})$, cell $(9, \mathrm{~W})$, and cell $(9, \mathrm{Z})$ are the standard deviation of demand. Rows 11 13: columns $\mathrm{R}$ to $\mathrm{Z}$ calculate red zone safety using the equation (7) for E-SS and (19) for P-SS, respectively, and calculate $\sigma_{L}$ using the equation (2) for T-SS. Rows 15 17: columns $\mathrm{R}$ to $\mathrm{Z}$ calculate red zone as the sum of red zone base and red zone safety and as multiplying by $\sigma_{L}$ and $z=5$ in cell $(12, \mathrm{P})$ for T-SS. Rows 19 21: columns $\mathrm{R}$ to $\mathrm{Z}$ calculate top of yellow as the sum of red zone and yellow zone. Rows 23 25: columns $\mathrm{R}$ to $\mathrm{Z}$ calculate top of green as the sum of red zone, yellow zone, and MOQ. Rows 27 29: columns $\mathrm{R}$ to $\mathrm{Z}$ store the average inventory from the simulation. Rows $31 \sim 33$ : columns $\mathrm{R}$ to $\mathrm{Z}$ store the average shortage quantity from the simulation. Rows 35 37: columns $\mathrm{R}$ to $\mathrm{Z}$ store the number of stockouts from the simulation. Cell $(2, \mathrm{H})$ : the average daily demand. Cell $(3, \mathrm{H})$ : the annual average demand obtained by multiplying 365 days to cell $(2, \mathrm{H})$ (but not used in this simulation). Cell $(5, \mathrm{H})$ : standard deviation of the daily demand. Clicking row 1 (column R, S, or T) button will import row 9 (T). Clicking row 1 (column $\mathrm{U}, \mathrm{V}$, or $\mathrm{W}$ ) button will import row $9(\mathrm{~W})$. Clicking row 1 (column $\mathrm{X}, \mathrm{Y}$, or Z) button will import row $9(\mathrm{Z})$. Cell $(6, \mathrm{H})$ ) clicking row 1 (from $\mathrm{R}$ to $\mathrm{Z}$ ) will show the corresponding value in $\mathrm{R}$ to $\mathrm{Z}$ (not used in the simulation). Cell $(7, \mathrm{H})$ : minimum order quantity (2 days' average demand). Cell $(8, \mathrm{H})$ : order spike threshold set to be $50 \%$ of the red zone. Cell $(10, \mathrm{H})$ : clicking row 1 (button $\mathrm{R}$ to $\mathrm{Z}$ ) imports the corresponding top of green ( $\mathrm{R}$ to $\mathrm{Z}$, rows 23-25). Cell (11, H): clicking row 1 (button $\mathrm{R}$ to $\mathrm{Z}$ ) imports the corresponding top of yellow ( $\mathrm{R}$ to $\mathrm{Z}$, rows 19-21). Cell $(12, \mathrm{H})$ : clicking row 1 (button $\mathrm{R}$ to $\mathrm{Z}$ ) imports the corresponding top of red ( $\mathrm{R}$ to $\mathrm{Z}$, rows 15-17). Cell $(13, \mathrm{H})$ : clicking row 1 (button $\mathrm{R}$ to $\mathrm{Z}$ ) imports the corresponding top of red zone base ( $\mathrm{R}$ to $\mathrm{Z}$, rows 7-9). Cell $(2, \mathrm{I})$ and cell $(4, \mathrm{I})$ : not used; cell $(2, \mathrm{~L})$ and cell $(2, \mathrm{~L})$ : not used; cell $(2, \mathrm{M})$ : shows the lead time $(5,20$, or 35$)$; cell $(3, M)$ and cell $(4, M)$ : not used. Macro "DemandCal": to apply the order spike, we assume that a daily demand comprises two demand orders. (1) Generate a daily demand from lognormal $(1,000, \mathrm{sd} 2)$. (2) Generate demand order 1 from lognormal (500, sd2). (3) Determine demand order 2 as daily demand-demand order 1. (4) The later shipping date is assigned to the larger order and the earlier shipping date to the smaller order. In row 11, five columns (AA AE) store boundary values of order quantity for $\mathrm{LT}=5$. In row 12 , twenty columns $(\mathrm{AA} \sim \mathrm{AT}$ ) store boundary values of order quantity for $\mathrm{LT}=20$. In row 13, 35 columns $(\mathrm{AA} \sim \mathrm{BI})$ store boundary values of order quantity for $\mathrm{LT}=35$. Row 22 to the last row store order quantity received on the day. Macro "DDMRP" row 21 stores the initial values of the received quantity on the day (column B), on-hand (column D), and available inventory as the size of yellow zone (column E). Rows 22 to 3,671 (for 10 times of 365 days): column B stores the received quantity on the day, column C stores daily demand, column D stores onhand inventory, column $\mathrm{E}$ stores open order, column $\mathrm{F}$ stores available inventory, column $G$ stores order to be replenished, column $\mathrm{H}$ stores lead time, and column I stores one order quantity. Simulation procedure is in sheets E-SS, T-SS, and P-SS. Based on each equation, red base, red safety, yellow zone, and green zone are calculated using Excel function formula before starting. (1) Enter one of the three LT values 5, 20, and 35 in cell $(2, M)$. (2) Click one of the nine buttons $(\mathrm{R} \sim \mathrm{Z})$, each of which indicates the combination of three demand variation levels and three LT variation levels. (3) Save. (4) Click DemandCal. (5) Click DDMRP. (6) Go to (2). (7) Go to (1). Sheet "Results compare" This sheet shows the comparison of the resulting inventory and stockouts stored in columns $\mathrm{R}$ to $\mathrm{Z}$ of rows 27 to 37 in the sheets E-SS, $\mathrm{T}-\mathrm{SS}$, and P-SS, respectively. The average inventory, average quantity of stockout, and the number of stockouts are compared and shown graphically. File (3): file simulation result-summary. Sheet 1: Tables 1 and 2 shown in the paper. Sheet 2: Table 3 shown in the paper. Sheet 3: to illustrate Figure 3 used in the paper. Column A: lead time 1 90. 
Column B: the values obtained by interpolating the lead time factor of DDMRP. Column C: the lead time multiplied by the interpolated value. Column D: square root value of the lead time. Column E: the value obtained by applying $1.02 \sqrt{ } \mathrm{L}+1.15$ from equation (16) (excluding average demand). Sheet 4: collection of results from two files (1) and (2). The average inventory and the number of stockouts are compared. Sheet 5: not used. (Supplementary Materials)

\section{References}

[1] E. A. Silver, D. F. Pyke, and R. Peterson, "Chapter 7: individual items with probabilistic demand," Inventory Management and Production Planning and Schedulingpp. 198-295, John Wiley \& Sons, Danver, MA, USA, 3th edition, 1998.

[2] R. H. Ballou and S. K. Srivastava, "Chapter 9 inventory policy decisions," in Business Logistics/Supply Chain Management, pp. 336-433, Pearson Education, Noida, India, 5th edition, 2004.

[3] G. R. Brown, Materials Management System, pp. 140-157, Krieger, Malabar, FL, USA, 1977.

[4] E. M. Goldratt, I. Eshkoli, and J. Brownleer, Isn't It Obvious, North River Press, Great Barrington, MA, USA, 2009.

[5] TOCICO, June 2018, https://www.tocico.org/page/ref_bank1.

[6] C. Ptak and C. Smith, Orlicky's Material Requirements Planning, McGraw-Hill Education, Columbus, OH, USA, 3rd edition, 2011.

[7] C. E. Clark, "Mathematical analysis of an inventory case," Operations Research, vol. 5, no. 5, pp. 627-643, 1957.

[8] C. Das, "Inventory control for log-normal demand," Computers and Operations Research, vol. 10, no. 3, pp. 267-276, 1983.

[9] B. R. Cobb, R. Rumí, and A. Salmerón, "Inventory management with log-normal demand per unit time," Computers \& Operations Research, vol. 40, no. 7, pp. 1842-1851, 2013.

[10] E. M. Goldratt and J. Cox, The Goal, North River Press, Great Barrington, MA, USA, 1984.

[11] E. Schragenheim, H. W. Dettmer, and J. W. Patterson, Supply Chain Management at Warp Speed Integrating the System from End to End, CRC Press Book, Boca Raton, FL, USA, 2009.

[12] J. F. Cox III, G. John, and J. G. Schleier Jr., Theory of Constraints Handbook, McGraw-Hill, New York, NY, USA, 2010.

[13] S. Bonatsos, Milk Run Replenishment in Cyprus, TOCICO, Albany, NY, USA, 2013.

[14] D. S. Han and O. K. Kwon, "The study on comparative analysis of replenishment-based and forecast-based inventory policies: case of a consumer electronics manufacturing company," Korea Logistics Society, vol. 22, no. 2, pp. 81-91, 2014.

[15] M. Christopher, "The agile supply chain," Industrial Marketing Management, vol. 29, no. 1, pp. 37-44, 2000.

[16] Wikipedia, June 2017, https://en.wikipedia.org/wiki/AMR_ Research.

[17] F. T. S. Chan, S. H. Chung, and S. Wadhwa, "A heuristic methodology for order distribution in a demand driven collaborative supply chain," International Journal of Production Research, vol. 42, no. 1, pp. 1-19, 2004.

[18] E. M. Goldratt, E. Schragenheim, and C. A. Ptak, Necessary But Not Sufficient, North River Press, Great Barrington, MA, USA, 2000.

[19] C. Ptak and C. Smith, Material Requirements Planning, pp. 151-157, Industrial Press, New York, NY, USA, 2016.
[20] G. R. Brown, Statistical Forecasting for Inventory Control, pp. 105-127, McGraw-Hill Book Company, New York, NY, USA, 1959.

[21] Demand Driven Institute, June 2017, http://www. demanddriveninstitute.com/case-studies.

[22] J. S. Lee and S. Y. Jang, "A system dynamics model to evaluate the performances of MRP and demand-driven MRP," Journal of the Korean Society of Supply Chain Management, vol. 14, no. 2, pp. 125-136, 2014.

[23] M. Ihme, Interpreting and Applying Demand Driven MRP: A Case Study, Nottingham Trent University, Nottingham, UK, 2015.

[24] R. Miclo, F. Fontanili, M. Lauras, J. Lamothe, and B. Milian, "An empirical study of demand-driven MRP," in Proceedings of the Information Systems Logistics and Supply Chain 6th International Conference, Bordeaux, France, June 2016.

[25] M. J. Shofa and W. O. Widyarto, "Effective production control in an automotive industry: MRP vs. demand-driven MRP," American Institute of Physics, vol. 1855, no. 1, Article ID 020004, 2017.

[26] S.-C. Rim, J. Jiang, and C. J. Lee, "Strategic inventory positioning for MTO manufacturing using ASR lead time," in Logistics Operations, Supply Chain Management and Sustainability, EcoProduction (Environmental Issues in Logistics and Manufacturing), pp. 441-456, Springer, Berlin, Germany, 2014.

[27] C. J. Lee and S. C. Rim, "Safety stock performance simulation for DDMRP replenishment," in Conference Proceedings of the Korean Society of Supply Chain Management, Seoul, South Korea, May 2018.

[28] P. R. Tadikamalla, "The lognormal approximation to the lead time demand in inventory control," Omega, vol. 7, no. 6, pp. 553-556, 1979. 


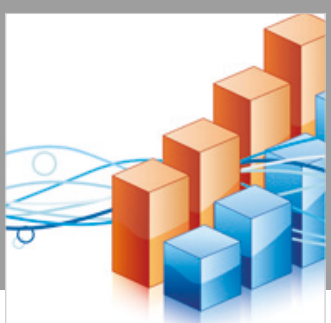

Advances in

Operations Research

\section{-n-m}
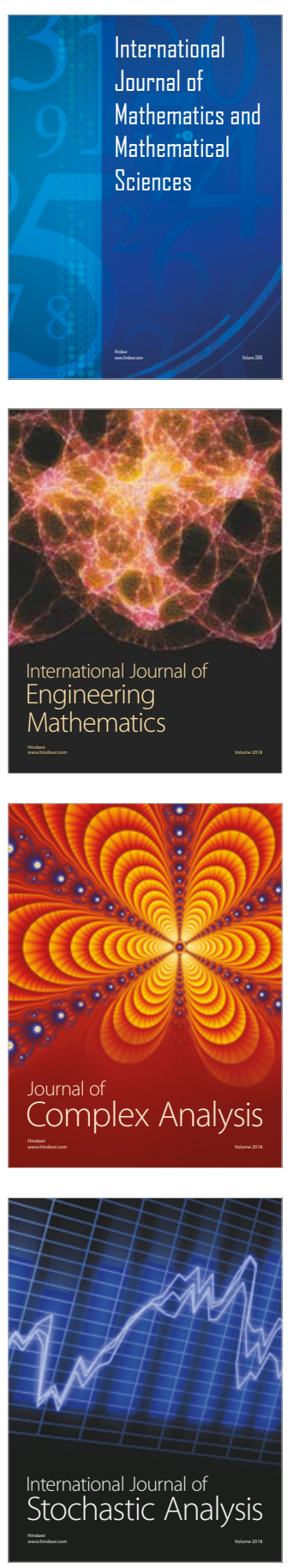
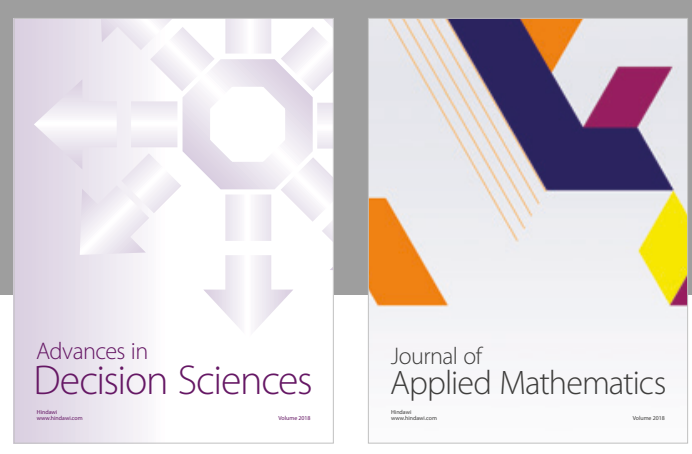

Journal of

Applied Mathematics
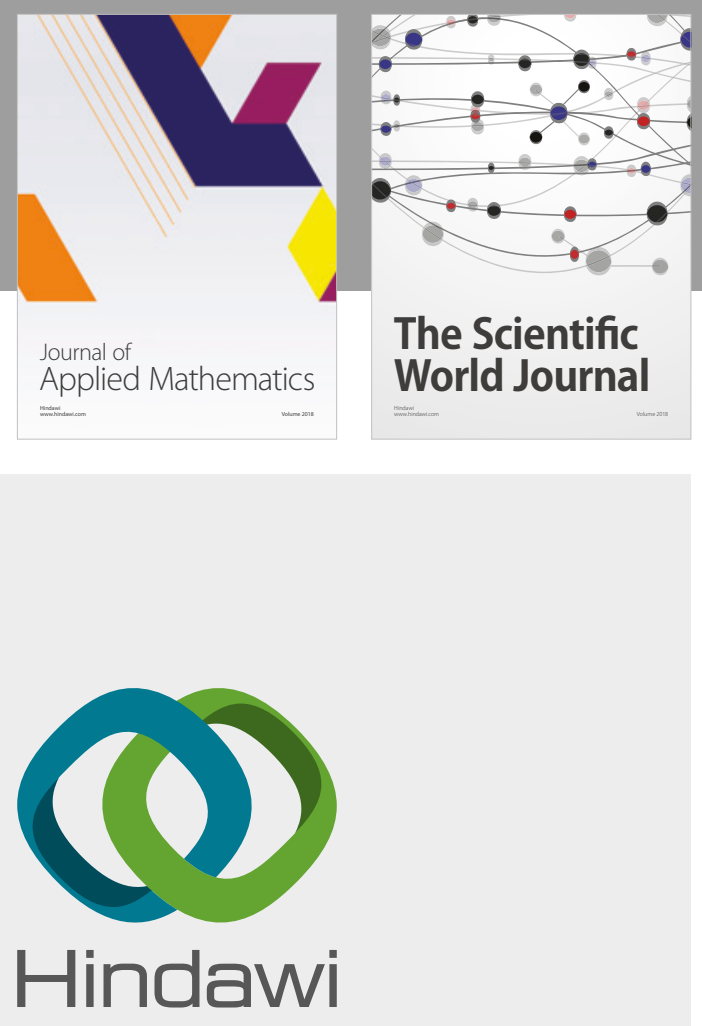

Submit your manuscripts at

www.hindawi.com

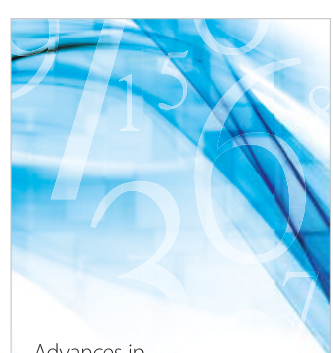

Advances in
Numerical Analysis
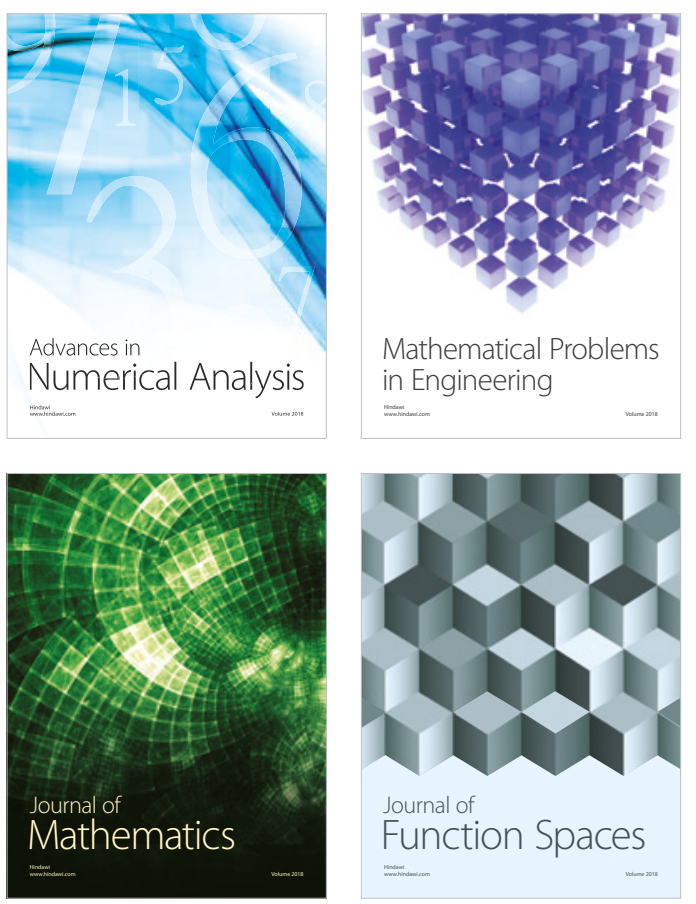

Mathematical Problems in Engineering

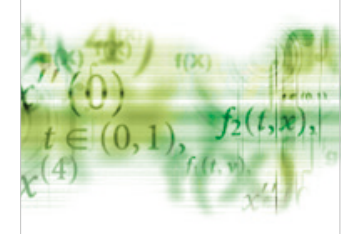

International Journal of

Differential Equations

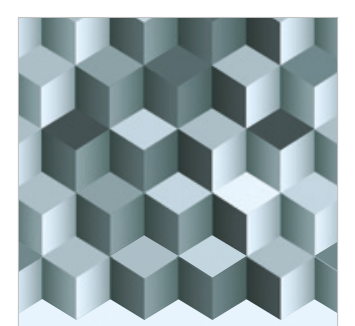

Journal of

Function Spaces

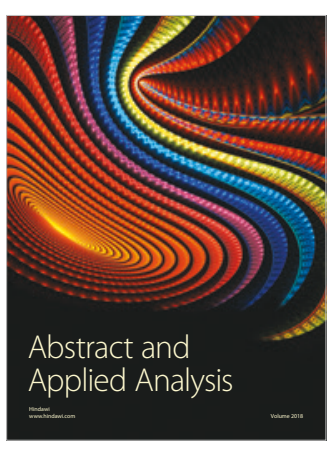

The Scientific

World Journal

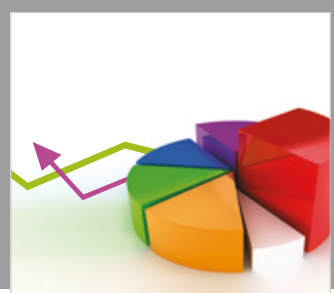

Journal of

Probability and Statistics
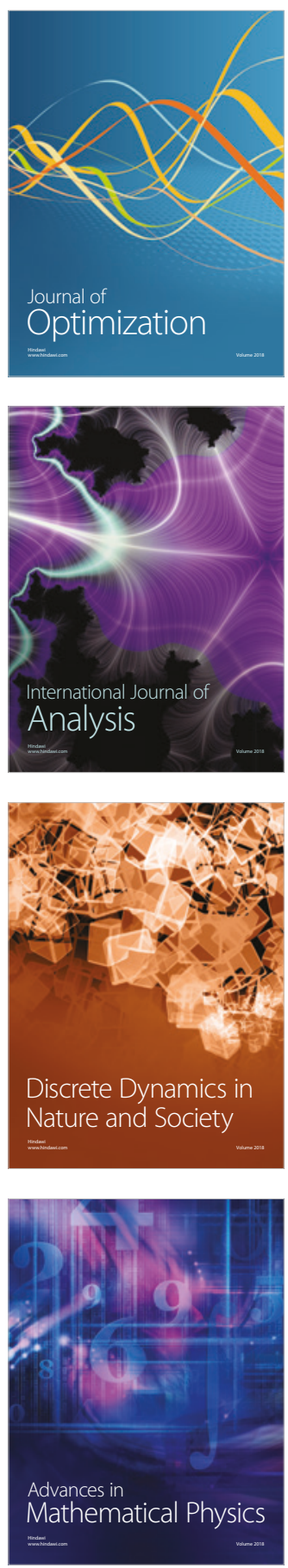